\title{
Continuous estimates of dynamic cerebral autoregulation: Influence of non-invasive arterial blood pressure measurements
}

\author{
RB Panerai ${ }^{1}$, EL Sammons ${ }^{2}$, SM Smith $^{1}$, WE Rathbone ${ }^{1}$, S Bentley ${ }^{3}$, \\ JF Potter ${ }^{4}$ and NJ Samani ${ }^{1}$ \\ ${ }^{1}$ Department of Cardiovascular Sciences, University of Leicester, \\ Leicester, UK \\ ${ }^{2}$ Faculty of Medicine, University of Leicester, UK \\ ${ }^{3}$ Department of Medical Physics, University Hospitals of Leicester NHS \\ Trust, UK \\ ${ }^{4}$ School of Medicine, University of East Anglia, UK \\ E-mail: rp9@le.ac.uk
}

$\begin{array}{ll}\text { Corresponding author: } & \text { RB Panerai } \\ & \text { Department of Medical Physics } \\ & \text { Leicester Royal Infirmary } \\ & \text { Leicester LE1 5WW } \\ \text { Tel. +44-116-2585511 } \\ \text { Fax +44-116-2586070 } \\ \text { e-mail: rp9@le.ac.uk }\end{array}$ 


\section{Abstract}

Temporal variability of parameters which describe dynamic cerebral autoregulation (CA), usually quantified by the short-term relationship between arterial blood pressure (BP) and cerebral blood flow velocity (CBFV), could result from continuous adjustments in physiological regulatory mechanisms, or could be the result of artefacts in methods of measurement, such as the use of non-invasive measurements of BP in the finger. In 27 subjects $(61 \pm 11$ years old $)$ undergoing coronary artery angioplasty, BP was continuously recorded at rest with the Finapres device and in the ascending aorta (Millar catheter, $\mathrm{BP}_{\mathrm{AO}}$ ), together with bilateral transcranial Doppler ultrasound in the middle cerebral artery, surface ECG and transcutaneous $\mathrm{CO}_{2}$. Dynamic CA was expressed by the autoregulation index (ARI), ranging from 0 (absence of CA) to 9 (best CA). Time-varying, continuous estimates of ARI $(A R I(t))$ were obtained with an auto-regressive moving-average (ARMA) model applied to a 60-s sliding data window. No significant differences were observed in the accuracy and precision of $A R I(t)$ between estimates derived from the Finapres and $\mathrm{BP}_{\mathrm{AO}}$. Highly significant correlations were obtained between $A R I(t)$ estimates from the right and left MCA (Finapres $\mathrm{r}=0.60 \pm 0.20 ; \mathrm{BP}_{\mathrm{AO}} \mathrm{r}=0.56 \pm 0.22$ ) and also between the $A R I(t)$ estimates from the Finapres and $\mathrm{BP}_{\mathrm{AO}}$ (right MCA $\mathrm{r}=0.70 \pm 0.22$; left MCA r $=0.74 \pm 0.22)$. Surrogate data showed that $A R I(t)$ was highly sensitive to the presence of noise in the CBFV signal, with both the bias and dispersion of estimates increasing for lower values of $A R I(t)$. This effect could explain the sudden drops of $A R I(t)$ to zero as reported previously. Simulated sudden changes in $A R I(t)$ can be detected by the Finapres, but the bias and variability of estimates also increase for lower values of ARI. In summary, the Finapres does not distort time-varying estimates of dynamic CA obtained with a sliding window combined with an ARMA model, but further research is needed to confirm these findings in healthy subjects and to assess the influence of different physiological maneuvers.

Keywords: non-stationarity, cerebral blood flow, physiological variability, mathematical model, non-invasive blood pressure 


\section{Introduction}

Under normal physiological conditions, blood flow to the brain is controlled by multiple mechanisms, including cerebral pressure-autoregulation (CA), which maintains cerebral blood flow (CBF) relatively constant for changes in arterial blood pressure (BP) in the range 60-150 mmHg (Paulson et al 1990). Early studies of CA adopted a 'static' approach, using mean values of BP and CBF averaged over several minutes. With the advent of transcranial Doppler ultrasound, it became possible to record $\mathrm{CBF}$ velocity (CBFV) with very high temporal resolution and this allowed the identification of transient responses of $\mathrm{CBFV}$ to sudden changes in $\mathrm{BP}$, giving rise to the concept of 'dynamic' CA (Aaslid et al 1989).

Dynamic CA has several advantages when compared to the static approach, mainly that it does not require the induction of long lasting changes in mean BP, usually achieved with the use of pharmacological agents (Panerai 1998). Although dynamic CA cannot be observed without changes in mean BP, relatively fast changes can be induced by several different manoeuvres such as the thigh cuff test (Aaslid et al 1989), Valsalva manoeuvre (Tiecks et al 1996), cold pressor test (Panerai et al 2001), synchronized breathing (Diehl et al 1995) or repeated squatting (Birch et al 1995). Of considerable interest is the possibility of using spontaneous fluctuations in BP and CBFV to model dynamic CA since this avoids any physiological perturbation and does not require any collaboration from the subject (Panerai 1998).

Despite our recent ability to record changes in CBFV with high temporal resolution, most approaches proposed to study dynamic CA have adopted a relatively low temporal resolution for the estimation of CA parameters. For estimates derived from spontaneous fluctuations in BP and CBFV, recordings usually last between 5-10 min. leading to a single set of CA parameters. One set of estimates is obtained from each manoeuvre performed to induce changes in BP, but each manoeuvre can take several minutes to perform (Czosnyka et al 1997, Panerai et al 1998, Panerai 1998, Tiecks et al 1995, Zhang et al 1998). There are two main reasons why it would be important to increase the temporal resolution of estimates of dynamic CA. Firstly, as with most physiological processes, it is unlikely that $\mathrm{CA}$ is a rigidly constant mechanism, but 
would rather show some intrinsic variability which can be modulated by other biological factors (Busija and Heistad 1984, Gotoh et al 1982, Jones et al 1995, Vern et al 1988). Secondly, because CA involves vasoactive responses and hence changes in cerebrovascular resistance, which physically relates $\mathrm{CBFV}$ to $\mathrm{BP}$, it cannot be regarded as a time-invariant system and will inevitably show some degree of nonstationarity. This is more likely to be manifested during physiological manoeuvres such as head-up tilt, exercise, Valsalva or lower-body negative pressure.

Previous studies of the variability or non-stationarity of dynamic CA involved analysis of spontaneous transients of BP (Panerai et al 2003a) or the use of a slidingwindow autoregressive-moving average (ARMA) approach (Panerai et al 2003b). Evidence of daily changes in the efficiency of CA in severe head injury patients was obtained with a sliding correlation index (Czosnyka et al 1997). Neural networks (Mitsis et al 2004), wavelet analysis (Latka et al 2005) and multimodal decomposition (Novak et al 2004) have also been proposed as techniques to cope with the non-stationarity of dynamic CA.

In a previous study, it was suggested that continuous non-invasive measurements of finger BP made with the Finapres (or Portapres) device might exacerbate the variability of dynamic CA parameters estimated during spontaneous fluctuations in BP and CBFV (Panerai et al 2003b). To test this hypothesis, continuous estimates of an index of dynamic CA (ARI) (Tiecks et al 1995) were obtained in patients undergoing coronary angioplasty, in whom BP was simultaneously recorded invasively (aorta) and non-invasively (finger). Surrogate data, generated by computer simulation, were used to validate the signal processing algorithms adopted to extract time-varying estimates of ARI.

\section{Methods}

\subsection{Subjects and measurements}

Recruited subjects were scheduled for routine elective percutaneous coronary interventions. Patients were excluded if they had any condition that could distort Finapres measurements of BP such as arthritis or Raynaud's disease. All patients were in a stable condition without evidence of significant valve disease. Ethical approval 
was obtained from the Leicestershire Ethics Committee and written informed consent was obtained before each study.

Patients were asked to fast for at least 6 hours before the procedure and also to avoid alcohol and nicotine. All studies were conducted in the morning in a temperature controlled recovery room, immediately following percutaneous coronary angiography or angioplasty. After the elective procedure, the angiographic catheter was replaced by a catheter-tip pressure transducer (Mikro-Tip SPC-454E, Millar Instruments, Houston, Texas), after it was zero-balanced. The Millar catheter was advanced in the ascending aorta with its final position $2-3 \mathrm{~cm}$ before the aortic valve checked by fluoroscopy. Cerebral blood flow velocity in the middle cerebral artery (MCA) was recorded bilaterally by Doppler ultrasound (2MHz, Scimed QVL842X, Bristol, UK) with probes placed over the temporal bone and secured with an adjustable head frame. The surface ECG was recorded with three standard chest leads and transcutaneous $\mathrm{CO}_{2}$ (TINA, Radiometer, Copenhagen, Denmark) was monitored continuously with an axillary probe overlying the Tail of Spence.The Finapres cuff (Finapres 2300, Ohmeda, Englewood, Colorado) of the appropriate size was placed around the middle or annular finger of the hand that was not cannulated and the hand was kept at atrial level. In most patients the hand was covered with a blanket to prevent it from getting cold. The Finapres cuff was repositioned until a stable waveform was achieved with the servo-adjust on, waveforms being considered stable when the period between servo-adjusts was $>30$ beats duration (Hope et al 2004). After all measurements were stable for 15 min., Finapres and Millar transducer calibration signals were recorded, followed by continuous data recording on digital audiotape (Sony PC 208AX, Tokyo, Japan) for two ten minute periods. A second calibration of the Finapres and Millar transducer was performed before the second recording. To avoid interruption of the recorded signals, the self-adjusting servo of the Finapres was switched off at the beginning of each recording.

\subsection{Data analysis}

Data were downloaded in real time onto a dedicated personal computer. An FFT was used to convert the Doppler signals into maximum frequency velocity envelopes with a $5 \mathrm{~ms}$ window resolution. The two BP signals, ECG and transcutaneous $\mathrm{CO}_{2}$ were 
sampled at 200 samples/s per channel. The data were visually inspected to select recordings that had good quality waveforms for both CBFV channels, as well as BP from the Finapres $\left(\mathrm{BP}_{\mathrm{FIN}}\right)$ and aorta $\left(\mathrm{BP}_{\mathrm{AO}}\right)$ uninterrupted for at least $7 \mathrm{~min}$. In patients who had two good quality recordings, only the longest recording was used for analysis. The CBFV signal was filtered with a 5-point median filter and all signals were low-pass filtered with a zero-phase eighth-order Butterworth digital filter with a cut-off frequency of $20 \mathrm{~Hz}$. Each cardiac cycle was automatically marked to determine the R-R interval from the ECG tracing. Heart rate was calculated from the R-R interval. For all available cardiac cycles in each recording, spline interpolation was used to resample the data with a time interval $0.2 \mathrm{~s}$ to create a uniform time base.

The autoregulation index (ARI) proposed by Tiecks et al (1995) was used to simulate and also to identify the temporal variability of CA. The Finapres or intra-aortic mean BP signal, represented generically by $P(t)$, was normalized:

$$
d P(t)=\frac{P(t)-P_{M}}{P_{M}-C r C P}
$$

where $P_{M}$ is the mean value of $P(t)$ for the data window. $C r C P$ is the critical closing pressure, and was chosen as $12 \mathrm{mmHg}$ (Tiecks et al 1995). The model predicted velocity, $V_{T}(t)$ is given by

$$
V_{T}(t)=V_{M}\left(1+d P(t)-K \cdot x_{2}(t)\right.
$$

where $\mathrm{K}$ is a parameter reflecting autoregulatory gain and $V_{M}$ is the mean velocity for the entire record. $x_{1}(t)$ and $x_{2}(t)$ are intermediate variables given by:

$$
\begin{aligned}
& x_{1}(t)=x_{1}(t-1)+\frac{d P(t)-x_{2}(t-1)}{f \cdot T} \\
& x_{2}(t)=x_{2}(t-1)+\frac{x_{1}(t)-2 \cdot D \cdot x_{2}(t-1)}{f \cdot T}
\end{aligned}
$$


$f, D$, and $T$ represent the sampling frequency, damping factor, and time constant parameters, respectively. Specific combinations of values of $K, D$, and $T$ were used to generate 10 grades of dynamic autoregulation, expressed by the ARI, ranging from 0 (absence of autoregulation) to 9 (best autoregulation) (Tiecks et al 1995).

Tieck's model was initially proposed to represent the CBFV response to a negative step change in BP, but it has been shown that it can also fit the CBFV-BP dynamic relationship during spontaneous fluctuations in $\mathrm{BP}$ or other challenges such as the Valsalva manoeuvre or lower body negative pressure (Panerai et al 2001). To simulate CBFV signals with constant values of ARI, $P(t)$ is taken as the input variable and the values of $\mathrm{K}, \mathrm{D}, \mathrm{T}$, corresponding to the selected value of ARI, were used with equations (2-4) to generate the velocity signal. To simulate velocity signals with timevarying CA, the ARI was increased stepwisely from 0 to 9 , in steps to 3, each step lasting $120 \mathrm{~s}$ (Fig. 3). Finally, the simulated velocity signal $V_{S}(t)$ was given by:

$V_{S}(t)=V_{T}(t)+\eta(t)$

where $V_{T}(t)$ is given by equation (2) and $\eta(t)$ is band-filtered random noise obtained by low-pass filtering gaussian white noise with a cutoff frequency of $0.3 \mathrm{~Hz}$.

Given a pair of pressure and velocity signals, an ARMA model (Ljung and Soderstrom 1983) was used to calculate the velocity impulse and step responses as the first step to the estimation of the ARI index. In a previous study, the ARMA model was found to provide more reliable values of ARI than direct fitting ot Eqs. 1-4 to time-series of V(t) and $\mathrm{P}(\mathrm{t})$ (Panerai et al 2003b). This approach was used with both measured and simulated velocity signals, and for both $\mathrm{BP}_{\mathrm{FIN}}$ and $\mathrm{BP}_{\mathrm{AO}}$.

The general structure of these models is

$$
V(t)=\sum_{i=1}^{M} a_{i} V(t-i)+\sum_{j=0}^{Q-1} b_{j} P(t-j)
$$


where the $a_{i}$ and $b_{j}$ are real coefficients and $[M, Q]$ are the model orders (Ljung and Soderstrom 1983). For each data segment, the model coefficients were estimated by the least-square method. Previous studies have indicated that a [2,3] model was the most suitable for modeling dynamic CA (Liu et al 2003, Panerai et al 2003b).

After obtaining the best fit for a data window of duration $\mathrm{N}_{\mathrm{W}}$, the impulse and step responses were calculated from $a_{i}$ and $b_{j}$, and the velocity step response generated by the ARMA model was matched to the most appropriate of the 10 step responses given by Tieck's model, using the correlation coefficient, but taking into account only the first $N_{f i t}=12 \mathrm{~s}$ of the step response. The implications of this choice will be discussed later. Once a best-fit curve was selected, a parabolic interpolation was performed to estimate the value of ARI to one decimal place. To obtain time-varying, continuous values of ARI, a $N_{W}=60 \mathrm{~s}$ sliding window was used and sequential estimates were obtained at $0.6 \mathrm{~s}$ intervals. Due to the duration of the window, estimates of ARI could not be obtained for the first and last $30 \mathrm{~s}$ of each record. The corresponding time series was represented by $A R I(t)$.

Three separate studies were performed: i) Simulation of constant values of ARI, ii) simulation of step changes in $A R I(t)$, as a staircase varying from 0 to 9 in steps of 3 , and iii) estimation of $A R I(t)$ from real data to characterize its variability and the influence of the $\mathrm{BP}$ source, that is $\mathrm{BP}_{\mathrm{FIN}}$ and $\mathrm{BP}_{\mathrm{AO}}$. In simulation studies, the velocity signal-to-noise ratio $\left(\mathrm{SNR}_{\mathrm{V}}\right)$ was expressed in decibels, that is

$$
S N R_{V}=10 \log _{10}\left(\frac{\sigma_{V}^{2}}{{\sigma_{N}}^{2}}\right)
$$

where $\sigma_{V}{ }^{2}$ and $\sigma_{N}{ }^{2}$ are the velocity and noise variances, respectively. $\mathrm{BP}_{\mathrm{AO}}$ was used to generate $V_{T}(t)$ signals and corresponding values of $A R I(t)$ were extracted using both $\mathrm{BP}_{\mathrm{FIN}}$ and $\mathrm{BP}_{\mathrm{AO}}$ after noise was added to $V_{T}(t)$ (eq. 5). Only one simulated $V_{T}(t)$ signal was generated for each subject for each value of ARI. The appropriate $\mathrm{SNR}_{V}$ (eq. 7) was found by comparing the coherence function between $\mathrm{BP}_{\mathrm{AO}}$ and $\mathrm{V}_{\mathrm{S}}(\mathrm{t})$ with values of coherence between $\mathrm{CBFV}$ and invasive aortic BP previously found with measured data. At the frequency of $0.1 \mathrm{~Hz}$ this representative value of the squared coherence 
was chosen as $\gamma^{2}=0.65$ (Sammons et al 2007). For simulations and estimates derived from patient data, the continuous $A R I(t)$ was averaged for each subject $(\overline{A R I(t)})$ and the population mean \pm SD was calculated, as well as the intra-subject variability, represented by the standard deviation of $A R I(t)$ for each recording. With patient data,

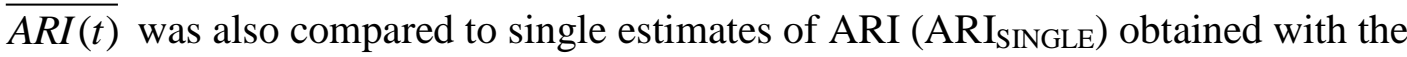
ARMA model using the entire data record.

\subsection{Statistics}

All comparisons used exactly the same number of cardiac cycles extracted from the Finapres and intra-aortic measurements. Differences between estimates of ARI were tested with Student's paired t-test. Differences between intra-subject variabilities were tested with the F-test for the ratio of two variances. Differences between probability distributions were tested with the $\chi^{2}$ test. Pearson correlation coefficients were also calculated and tested with the log transformation followed by the Z-test. Linear regressions between intra-subject coefficients of variation $(\mathrm{CoV})$ of $\mathrm{ARI}(\mathrm{t})$ and $\mathrm{CoV}$ $\mathrm{BP}$ were performed to test for the influence of $\mathrm{BP}$ variability on the dispersion of $A R I(t)$ values. A value of $\mathrm{p}<0.05$ was considered significant.

\section{Results}

A total of 45 patients were recruited, but good quality Doppler recordings for both MCAs were only obtained in 27 subjects. The main reason for rejection was the absence of a suitable acoustic window in one or both sides. Table 1 gives the demographic and baseline characteristics of the population studied.

\subsection{Simulation study}

In simulations with fixed values of $A R I$, reducing the $\mathrm{SNR}_{\mathrm{V}}$ led to increases in $\mathrm{ARI}_{\mathrm{AO}}$ bias and also in intra-record variability as expressed by the CoV in Fig. 1. These changes were highly dependent on the value of ARI selected. The highest bias and $\mathrm{CoV}$ were observed for $\mathrm{ARI}=0$ and the smallest for $\mathrm{ARI}=9$ (Fig. 1). For $\mathrm{ARI}=5$, the bias remained relatively stable between 0 and $10 \mathrm{~dB}$ and the $\mathrm{CV}$ tended to show an asymptotic behaviour for higher values of $\mathrm{SNR}_{V}$ (Fig. 1). The population 
cumulative probability distributions for the continuous estimates of ARI for these fixed-value simulations are given in Fig. 5A.

For $\mathrm{ARI}=5$, the coherence criterion of $\gamma^{2}(0.1 \mathrm{~Hz})=0.65$ was met for simulations with $\mathrm{SNR}_{\mathrm{V}}=3 \mathrm{~dB}$. The corresponding mean $\pm \mathrm{SD}$ values of coherence for simulations with $\mathrm{SNR}_{\mathrm{V}}=0 \mathrm{~dB}$ and $10 \mathrm{~dB}$ were $\gamma^{2}=0.56 \pm 0.15$ and $0.88 \pm 0.09$, respectively. Figure 2 shows mean $\pm \mathrm{SD}$ of $\overline{A R I(t)}$ and intra-subject variability for simulations with fixed values of $\mathrm{ARI}$ for $\mathrm{SNR}_{\mathrm{V}}=3 \mathrm{~dB}$. In general, $\mathrm{ARI}_{\mathrm{FIN}}$ was above and $\mathrm{ARI}_{\mathrm{AO}}$ was below the reference value of ARI. Mean values of $\mathrm{ARI}_{\mathrm{FIN}}$ were significantly greater than corresponding values derived from aortic pressure for reference ARI $\leq 8(p<0.05)$. The bias increased as the reference value of ARI was reduced. The intra-subject variability was significantly greater for $A R I_{F I N}$ in relation to $A R I_{A O}$ for reference ARI $\leq 3(p<0.05)$. Both estimates of ARI showed reduced variabilities at higher values of reference ARI (Fig. 1, bottom).

Figure 3 represents the population ensemble average $A R I(t)$ for the time-varying staircase simulation, together with representative data from a 65 year-old male subject. Both mean $A R I_{F I N}(t)$ and mean $A R I_{A O}(t)$ showed considerable differences in relation to the correct value of $\mathrm{ARI}$, but for $A R I_{A O}(t)$ the error decreases for higher values of ARI. For simulations with $\mathrm{SNR}_{\mathrm{V}} \geq 20 \mathrm{~dB}, A R I_{A O}(t)$ followed the correct value with much greater accuracy, but the $A R I_{F I N}(t)$ error remained approximately the same. At each transition of the reference $A R I(t)$ staircase, there was a slow response for both estimates of $A R I(t)$, due to the duration of the estimation window which was $60 \mathrm{~s}$ in all cases (Fig. 3B).

\subsection{Patient data}

Representative estimates of $A R I(t)$ are given in Fig. 4 for both MCAs. Most subjects showed wide variations in $A R I(t)$, often including values of $A R I=0$ and $A R I=9$ in the same segment of data as shown in Fig. 4E and 4F. For the population as a whole, intra-subject mean values of $A R I(t)$ were not significantly different from estimates from the entire record (Table 2), but the intra-subject variability was considerable, as expressed by the $\mathrm{CoV}$ in Table 2 . ARI $\mathrm{AIN}_{\mathrm{N}}$ was significantly higher than $\mathrm{ARI}_{\mathrm{AO}}$ for both MCAs (right $\mathrm{p}<0.05$; left $\mathrm{p}<0.01$ ). Figure 5B complements the information in 
Table 2 by showing the population cumulative probability distribution for $A R I(t)$. Noteworthy, estimates derived from both the Finapres and intra-aortic pressure had very similar incidence of values in the [0-0.5] bin range.

Highly significant correlation coefficients $(\mathrm{p}<0.001)$ were obtained between $A R I(t)$ estimates from the right and left MCA for both $\mathrm{ARI}_{\mathrm{FIN}}$ and $\mathrm{ARI}_{\mathrm{AO}}$, respectively $0.60 \pm$ 0.20 and $0.56 \pm 0.22$. Likewise, there was good correlation between the invasive and non-invasive estimates for each MCA side, corresponding to $0.70 \pm 0.21$ for the right MCA and $0.74 \pm 0.22$ for the left MCA. A sensitivity analysis performed with values of $N_{W}$ above and below $60 \mathrm{~s}$ and values of $N_{f i t}$ above and below $12 \mathrm{~s}$ led to slightly smaller correlation values in comparison with the values above. On the other hand, reducing $N_{f i t}$ to 6 s reduced the coefficient of variation (Table 2) by approximately $40 \%$ (data not shown).

Linear regressions between intra-subject $\mathrm{CoV}$ of $\mathrm{ARI}(\mathrm{t})$ for both MCAs and $\mathrm{CoV}$ of $\mathrm{BP}$ were not significant for either $\mathrm{BP}_{\mathrm{AO}}$ or $\mathrm{BP}_{\mathrm{FIN}}$. 


\section{Discussion}

\subsection{Influence of the Finapres}

In a previous study, estimates of dynamic CA obtained with the Finapres were shown to produce single values of ARI that were greater than corresponding measures derived from intra-aortic BP recordings, although the differences were relatively small (Sammons et al 2007). However, there were reasons to suspect that the Finapres could lead to much greater differences in continuous estimates of dynamic CA. First, reducing the data window for estimating parameters like the ARI is bound to increase the variability of estimates and their bias. Second, previous attempts to extract continuous estimates of $\mathrm{ARI}$, using only the $\mathrm{BP}_{\mathrm{FIN}}$ as the input signal, showed that sudden drops of $A R I(t)$ were a fairly frequent occurrence and led to the hypothesis that such nonphysiological behaviour could be caused by temporary malfunction of the Finapres (Panerai et al 2003b). Finally, another recent study showed that despite a null-bias between the Finapres and intra-aortic BP, relatively large transient differences in systolic, diastolic and mean BP can occur, lasting approximately $20 \mathrm{~s}$ (Panerai et al 2007). These transient drifts of the Finapres could then lead to much larger localized differences in $A R I(t)$ when compared to estimates derived from the

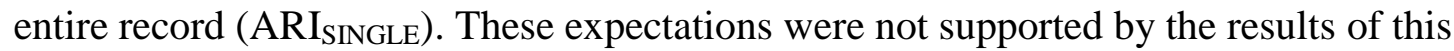
investigation. In the simulations (Figs $2 \& 3$ ), ARI FIN was significantly higher that $\mathrm{ARI}_{\mathrm{AO}}$, but this could have been due to using $\mathrm{BP}_{\mathrm{AO}}$ as the reference signal with added noise. Nevertheless, the differences were not greater than previously observed for estimates derived from the entire record (Sammons et al 2007) using real patient data. Likewise, both the intra-subject variability and the CoV did not show significant differences between $\mathrm{BP}_{\mathrm{FIN}}$ and $\mathrm{BP}_{\mathrm{AO}}$ estimates (Table 2) and the correlation coefficients between right and left MCA estimates of $A R I(t)$ were not significantly different from those for $\mathrm{ARI}_{\mathrm{AO}}$, either. As shown in Fig. 5B, the probability distributions of $A R I_{F I N}$ and $\mathrm{ARI}_{\mathrm{AO}}$ were similar and the number of 'crashes' to $\mathrm{ARI}=$ 0 (Figs. 4E \& 4F) were also equivalent as shown by the $\%$ of values for ARI $<0.5$.

\subsection{Physiological significance of short-term changes in cerebral autoregulation}

The conclusion that the Finapres does not distort continuous estimates of ARI, in comparison with values extracted with $\mathrm{BP}_{\mathrm{AO}}$, provides reassurance about the validity 
of previous studies of the longitudinal variability of dynamic CA, that were performed using only the Finapres (Chen et al 2006, Giller and Mueller 2003, Latka et al 2005, Liu et al 2005, Mitsis et al 2004, Panerai et al 2003a, 2003b). The problem of validating methods to describe the non-stationarity of dynamic CA can be appreciated when it is considered that a 'gold standard' for dynamic CA does not exist, even under the assumption that CA does not show short-term variability (Panerai 1998). Under these circumstances, the use of surrogate data can provide some indication about the reliability of time-varying estimates of dynamic CA. In other words, given the patterns of variability exemplified by the subject in Fig. 4, to what extent can we trust that such wide short-term changes are truly physiological? If that is not the case, then what is being observed is the result of inappropriate methods (e.g. ARMA + ARI), noise, or a combination of both.

In the absence of noise, simulations with a fixed value of ARI showed a negligible bias for estimates of $A R I(t)$ obtained with the ARI model fitted to the CBFV step response obtained with the ARMA method (Fig. 1). Although Simpson et al (2004) showed that the Tiecks model discriminates poorly between different values of ARI for estimates derived from spontaneous fluctuations in $\mathrm{BP}$ and $\mathrm{CBFV}$, this problem should be much attenuated by first calculating the CBFV step response therefore avoiding the application of equations (1-4) directly to the data (Panerai et al 2003b). Thus, accepting that the estimation of ARI by means of the CBFV step response is an unbiased and consistent estimator as shown by the results in Fig. 1, the next question is to assess its robustness to noise. As shown by Figs. 1 and 2, the influence of noise is dependent on the value of ARI. It is not entirely clear why the bias for ARI $=0$ is much higher than corresponding values for $\mathrm{ARI}=9$, given that both are in the extremes of the closed interval $[0,9]$. On the other hand, it is understandable that the $\mathrm{CoV}$ for $\mathrm{ARI}=0$ should be the highest (Fig. 1B) since the denominator would tend to be lower than for other values of $A R I$. Despite reducing the $S R_{V}$ to $0 \mathrm{~dB}$, the bias observed for values of $\mathrm{ARI}=5$ remained relatively low and the $\mathrm{CV}$ reached a maximum of $40 \%$.

Although the true $\mathrm{SNR}_{\mathrm{V}}$ of CBFV is not known, an approximation can be derived from the coherence function between BP and CBFV. Several research groups have shown that the coherence tends to be less than 0.5 at very low frequencies (VLF < 
$0.07 \mathrm{~Hz}$ ), rising above 0.5 at higher frequencies (>0.1 Hz) (Panerai et al 1998, Panerai et al 2006, Zhang et al 1998). The low coherence at VLF has been attributed to the non-linear relationship between CBFV and BP due to changes in CVR (Panerai et al 2006). For this reason the VLF band is not suitable for estimating the $\mathrm{SNR}_{V}$, but this problem should not exist at higher frequencies where dynamic CA is not as effective. Unfortunately, the limitation in this case is the relatively low amount of spectral power in the BP and CBFV signals which would make estimates less reliable at higher frequencies. As a compromise, the coherence at $0.1 \mathrm{~Hz}$ was chosen to identify the equivalent level of band-filtered noise to be added to the simulated, noise-free velocity signal generated with eqs. (1-4). Using previously reported values of coherence between $\mathrm{BP}_{\mathrm{AO}}$ and $\mathrm{CBFV}$, the appropriate $\mathrm{SNR}_{\mathrm{V}}$ was found to be $3 \mathrm{~dB}$. Noteworthy, this is a worst case condition because the true coherence between $\mathrm{BP}_{\mathrm{AO}}$ and CBFV is likely to be higher than 0.65 when the contribution of CVR (or RAP resistance-area product) is taken into account (Panerai et al 2006). Performing realistic simulations with $\mathrm{SNR}_{\mathrm{V}}=3 \mathrm{~dB}$ confirmed the dependence of the bias and variability on the simulated fixed value of ARI (Fig. 2). Moreover, the corresponding probability distributions (Fig. 5A) suggest that sudden drops to ARI $=0$ (Figs. 4E \& $4 \mathrm{~F}$ ) do not tend to occur for values of $\mathrm{ARI} \geq 4$. By interpolation, the equivalent value of fixed ARI that would lead to the same incidence of zero values as in the real data (Fig. 5B) would be ARI = 2.3. One possible interpretation, is that sudden drops, or 'crashes' to ARI = 0 can take place, due to the presence of noise, when the true physiological ARI approaches values $\leq 3$.

The evidence provided by simulation studies, that short-term variability in continuous estimates of dynamic CA reflect true physiological changes, is supported by previous reports in the literature. Czosnyka et al (1997) adopted a correlation index between BP and ICP (PRx) with a moving window of $200 \mathrm{~s}$. They observed both short-term and day-to-day changes in CA in head injury patients, but some of those changes could have been caused by physiological instability in critically ill patients. Under more stable physiological conditions, Mitsis et al (2004) showed longitudinal fluctuations in frequency-domain estimates of multivariate, non-linear, first- and second-order kernels for the effects of $\mathrm{BP}$ and $\mathrm{PaCO}_{2}$ on $\mathrm{CBFV}$, using a sliding window of 6 min. duration. Using the phase difference between CBFV and BP, as an indicator of dynamic CA (Birch et al 1995, Diehl et al 1995), Latka et al (2005) 
performed wavelet analysis to obtain time-varying changes in phase which showed very rapid fluctuations, similarly to that we observed with $A R I(t)$. Instantaneous changes in phase were also reported by Chen et al (2006), using a Hilbert transform approach. Large intra-subject variability in phase could be observed in healthy subjects at rest (their Fig. 3c), but unfortunately $\mathrm{CoV}$ or other measures of variability were not presented. Giller and Mueller (2003) performed time-frequency analysis showing an intermittent agreement between BP and CBFV in healthy subjects. These findings let the authors to conclude, in their own words, that "autoregulation is impaired intermittently in normal subjects, challenging the belief that autoregulation is normally invariant and stable". Finally, to avoid the interference of any system identification methods, large spontaneous transients in BP were compared to corresponding transients in CBFV. Dramatic differences in temporal patterns also suggested that dynamic CA can present major longitudinal changes in healthy individuals (Panerai et al 2003a). Despite the need to replicate these isolated findings, in this case the lack of standardization can be seen as beneficial since it shows that short-term variability of dynamic CA is manifested through a diversity of methodological approaches.

In summary, continuous estimates of dynamic CA seem to reflect spontaneous fluctuations of autoregulatory mechanisms at rest, but considerable more work is needed to shed light on the physiological processes involved and to improve and standardize analytical methods.

\subsection{Limitations of the study}

Doppler ultrasound measurements of CBFV will only reflect changes in CBF if the diameter of the insonated artery (i.e. MCA) remains constant. Several previous studies have failed to demonstrate that major changes in MCA diameter can take place during recordings at rest, even in situations involving large changes in $\mathrm{BP}$ and $\mathrm{PaCO}_{2}$ (Newell et al 1994, Serrador et al 2000). Nevertheless, changes in MCA diameter could modulate the CBFV beat-to-beat signal and contribute to the short-term variability of $A R I(t)$ or other continuous measures of dynamic CA. This possibility is not very likely though, because dynamic CA is mainly manifested through temporal characteristics of beat-to-beat patterns of $\mathrm{CBFV}$, such as the phase lead in relation to $\mathrm{BP}$, rather than its absolute amplitude. 
The use of spontaneous BP fluctuations to obtain estimates of dynamic CA can only produce reliable results if there is enough 'input power', or BP variability in the first instance (Liu et al 2005, Panerai et al 2001, Panerai et al 2006, Simpson et al 2004, Zhang et al 1998)). ABP variability is thought to decrease with ageing and for this reason, given the mean age of our population (Table 1), it could lead to unreliable estimates of $\mathrm{ARI}(\mathrm{t})$ in our case, to the extent of producing the sudden drops in $A R I(t)$ shown in Figs. 4 and 5. There are several reasons why this possibility was unlikely. First, as shown in Fig. 4, during the sudden drops in $A R I(t)$, ABP variability is no different from other periods when $A R I(t)$ was more stable. We compared the CoV of ABP of our subjects with data from previous studies which included a wider range of ages (Panerai et al 2001, 2003a, 2003b, 2006) and found similar distributions for the $\mathrm{CoV}$ of BP. Finally, linear regression analyses of the intra-subject variability of $A R I(t)$ as a function of BP variability $(\mathrm{CoV})$ were not significant and recordings with elevated dispersion of $A R I(t)$ values occurred for cases with low, medium and high BP variability.

For ethical reasons, measurement of intra-aortic BP was only possible in patients undergoing catheterization for clinical indications. The subjects we studied had coronary artery disease and were likely to have an increased probability of having both extracranial and intracranial atherosclerotic disease. For the study of the influence of estimates derived from $\mathrm{BP}_{\mathrm{FIN}}$, in comparison with corresponding measures obtained from $\mathrm{BP}_{\mathrm{AO}}$, each subject acted as his/her own control and the levels of agreement observed suggest that their upper peripheral circulation was free from severe occlusive disease. Their mean values of ARI (Table 2) were also in good agreement with other studies based on healthy subjects, which seems to suggest the absence of severe carotid artery disease (Panerai et al 2001, Tiecks et al 1995, White and Markus 1997). Future studies are needed to compare the short-term variability of $A R I(t)$ found in this investigation with that of healthy individuals and patients with cerebrovascular diseases such as stroke or intracranial hypertension.

More work is also required on several methodological aspects of the approach adopted to quantify the temporal evolution of dynamic CA. Simulations of the influence of additive noise using random band-pass gaussian noise are only an 
approximation since the true nature and pathways of other physiological influences, such as fluctuations in $\mathrm{PaCO}_{2}$, which would behave as 'noise' in the identification of the relationship between CBFV and BP, are not known. A moving window of $60 \mathrm{~s}$ duration affords 100 data points with sampling interval of $0.6 \mathrm{~s}$ which should be enough to provide reliable estimates of $A R I(t)$ when it is taken into account that the original proposal to derive the ARI from thigh cuff manoeuvres was based on a $30 \mathrm{~s}$ data window (Tiecks et al 1995). Our initial tests did not show significant improvement or deterioration when $\mathrm{N}_{\mathrm{W}}$ was changed to $120 \mathrm{~s}$ or $30 \mathrm{~s}$. One obvious consequence of the choice of $\mathrm{N}_{\mathrm{W}}$ though is the temporal resolution of $A R I(t)$ estimates as shown in Fig. 3B. For bedside monitoring, this time delay is unlikely to be a serious problem, but shorter time windows might be beneficial in studies involving interventions such as head-up tilt, sit-to-stand manoeuver or exercise. The data window was centralized at the current sample for offline analysis (Fig. 3) but in real time applications only past samples could be used and the time delay will shift accordingly. Finally, the choice of ARI as a measure of dynamic CA is not critical either since other parameters like the CBFV-BP phase difference of the CBFV step response would be equally valid. The advantages of using the ARI though, are a previously validated scale (Tiecks et al 1995) and its popularity in clinical studies of dynamic CA (Dawson et al 2000, Panerai et al 2004, White and Markus 1997).

\section{Conclusions}

Continuous estimates of dynamic CA obtained from noninvasive measurements of beat-to-beat BP in the finger are not substantially different from corresponding values derived from intra-aortic BP recordings. Surrogate data showed that a moving window ARMA method combined with the estimation of an index of dynamic CA (ARI) can reproduce step changes in CA, but the presence of noise in the velocity signal can lead to increasing bias and variability of estimates, mainly at low values of ARI. Further research is needed on the physiology of short-term variability of autoregulatory mechanisms and also on the suitability of different techniques for analysis of short-term fluctuations in healthy subjects and patients with cerebrovascular disease. 


\section{Acknowledgements}

We are grateful to Professor David H. Evans and Dr. Lingke Fan for development of the Doppler analyser software. This work was supported by the UK Engineering and Physical Research Council Grant EP/C001656/1.

\section{References}

Aaslid R, Lindegaard KF, Sorteberg W and Nornes H 1989 Cerebral autoregulation dynamics in humans Stroke $\mathbf{2 0} 45-52$

Birch AA, Dirnhuber MJ, Hartley-Davies R, Iannotti F and Neil-Dwyer G 1995 Assessment of autoregulation by means of periodic changes in blood pressure Stroke 26 834-8371995

Busija DW and Heistad DD 1984 Factors involved in the physiological regulation of the cerebral circulation Rev. Physiol. Biochem. Pharm. 101 162-211

Chen Z, Hu K, Stanley HE, Novak V and Ivanov PC 2006 Cross-correlation of instantaneous phase increments in pressure-flow fluctuations: applications to cerebral autoregulation Phys. Rev. E Stat. Nonlin. Soft Matter Phys. 73031915 Epub 2006 March 15

Czosnyka M, Smielewski P, Kirkpatrick P, Laing RJ, Menon D and Pickard JD 1997 Continuous assessment of the cerebral vasomotor reactivity in head injury Neurosurgery 41 11-19 
Dawson SL, Blake MJ, Panerai RB and Potter JF 2000 Dynamic but not static cerebral autoregulation is impaired in acute ischaemic stroke Cerebrovasc. Dis. $10126-132$

Diehl RR, Linden D, Lucke D and Berlit P 1995 Phase relationship between cerebral blood flow velocity and blood pressure. A clinical test of autoregulation Stroke 26 1801-1804

Giller CA and Mueller M 2003 Linearity and non-linearity in cerebral hemodynamics Med. Eng. \& Phys. 25 633-646

Gotoh F, Fukuuchi Y, Okayasu H, Tanaka K, Suzuki N and Kobari M 1982 Rhythmic changes in diameter of pial vessels and function fo autonomic nervous system. In: Cerebral Blood Flow: Effects of Nerves and Neurotransmitters, edited by Heistad DD. Amsterdam: Elsevier North Holland p. 409-417.

Hope SA, Meredith IT, Cameron JD 2004 Effect of non-invasive calibration of radial waveform on error in transfer-function-derived central aortic waveform characteristics Clin. Sci. 107 205-211

Jones SC, Williams JL, Shea M, Easley KA and Wei D 1995 Cortical cerebral blood flow cycling: anesthesia and arterial pressure Am. J. Physiol. Heart Circ.

Physiol. 268 H569-H575 
Latka M, Turalska M, Glaubic-Latka M, Kolodziej W, Latka D, West BJ 2005 Phase dynamics in cerebral autoregulation Am. J. Physiol. Heart Circ. Physiol. 289 H2272-H2279

Liu J, Simpson DM and Allen R 2005 High spontaneous fluctuations in arterial blood pressure improves the assessment of cerebral autoregulation Physiol. Meas. 26 $725-741$

Liu Y, Birch AA and Allen R 2003 Dynamic cerebral autoregulation assessment using an ARX model: comparative study using step response and phase shift analysis Med. Eng. \& Phys. 25 647-653

Ljung L and Soderstrom T 1983 Theory and practice of recursive identification. Cambridge, Massachusetts: MIT Press

Mitsis G, Poulin MJ, Robbins PA and Marmarelis VZ 2004 Nonlinear modeling of the dynamic effects of arterial pressure and $\mathrm{CO} 2$ variations on cerebral blood flow in healthy humans IEEE Trans. Biomed. Eng. 51 1932-1943

Newell DW, Aaslid R, Lam A, Mayberg TS and Winn HR 1994 Comparison of flow and velocity during dynamic autoregulation testing in humans Stroke 25 793797

Novak V, Yang ACC, Lepicovsky L, Goldberger AL, Lipsitz LA and Peng CK 2004 Multimodal pressure-flow method to assess dynamics of cerebral autoregulation in stroke and hypertension BioMed. Eng. OnLine 339 
Panerai RB 1998 Assessment of cerebral pressure autoregulation in humans - a review of measurement methods Physiol. Meas. 19 305-338

Panerai RB, Carey BJ and Potter JF 2003a Short-term variability of cerebral blood flow velocity responses to arterial blood pressure transients Ultrasound Med. Biol. 29 31-38

Panerai RB, Dawson SL, Eames PJ and Potter JF 2001 Cerebral blood flow velocity response to induced and spontaneous sudden changes in arterial blood pressure Am. J. Physiol. Heart Circ. Physiol. 280 H2162-H2174

Panerai RB, Eames PJ and Potter JF 2003b Variability of time-domain indices of dynamic cerebral autoregulation Physiol. Meas. 24 367-381

Panerai RB, Eames PJ and Potter JF 2006 Multiple coherence of cerebral blood flow velocity in humans Am..J. Physiol. Heart Circ. Physiol. 291 H251-H259

Panerai RB, Kerins V, Fan L, Yeoman PM, Hope T and Evans DH 2004 Association between dynamic cerebral autoregulation and mortality in severe head injury Brit. J. Neurosurg. 18 471-479

Panerai RB, Rennie JM, Kelsall AWR and Evans DH 1998 Frequency-domain analysis of cerebral autoregulation from spontaneous fluctuations in arterial blood pressure Med. \& Biol. Eng. \& Comput. 36 315-322 
Panerai RB, Sammons EL, Rathbone WE, Bentley S, Potter JF and Samani NJ 2007 Transient drifts between Finapres and continuous intra-aortic measurements of arterial blood pressure Blood Pres. Monit. 12 369-376

Paulson OB, Strandgaard S and Edvinson L 1990 Cerebral autoregulation Cerebrovasc. Brain Metab. Reviews 2 161-192

Sammons EL, Samani NJ, Smith SM, Rathbone WE, Bentley S, Potter J and Panerai RB 2007 Influences of noninvasive peripheral arterial blood pressure measurements on assessment of dynamic cerebral autoregulation J. Applied Physiol. 103 369-375

Serrador JM, Picot PA, Rutt BK, Shoemaker JK and Bondar RL 2000 MRI measures of middle cerebral artery diameter in conscious humans during simulated orthostasis Stroke 31 1672-1678

Simpson DM, Panerai RB, Ramos EG, Lopes JMA, Marinatto MNV, Nadal J and Evans DH 2004 Assessing blood flow control through a bootstrap method. IEEE Trans. Biomed. Eng. 51 1284-1286

Tiecks FP, Douville C, Byrd S, Lam AM and Newell DW 1996 Evaluation of impaired cerebral autoregulation by the Valsalva maneuver Stroke 27 1177-1182

Tiecks FP, Lam AM, Aaslid R and Newell DW 1995 Comparison of static and dynamic cerebral autoregulation measurements Stroke 26 1014-1019 
Vern BA, Schuette WH, Leheta B, Juel VC and Radulovacki M 1988 Low-frequency oscillations of cortical oxidative metabolism in waking and sleep J. Cerebr. Blood Flow Metab. 8 215-226

White RP and Markus HS 1997 Impaired dynamic cerebral autoregulation in carotid artery stenosis Stroke $\mathbf{2 8}$ 1340-1344

Zhang R, Zuckerman JH, Giller CA and Levine BD 1998 Transfer function analysis of dynamic cerebral autoregulation in humans Am. J. Physiol. Heart Circ.

Physiol. 274 H233-H241 
Table 1. Demographics and baseline characteristics of the study population $(\mathrm{N}=27)$.

$\begin{array}{lc}\text { Parameter } & \text { Mean } \pm \text { SD or } \\ \text { number }\end{array}$


Table 2 - Population mean \pm SD of continuous and punctual estimates of ARI. ARI SINGLE $_{\text {S }}$ was calculated with $\mathrm{N}_{\mathrm{W}}$ equal the duration of the entire recording. (See Methods).

\begin{tabular}{|l|c|c|c|c|}
\hline \multirow{2}{*}{\multicolumn{1}{|c|}{ Parameter }} & \multicolumn{2}{c|}{ Intra-aortic pressure } & \multicolumn{2}{c|}{ Finapres } \\
\cline { 2 - 5 } & Right MCA & Left MCA & Right MCA & Left MCA \\
\hline ARI $_{\text {SINGLE }}$ & $4.95 \pm 2.27$ & $5.25 \pm 2.18$ & $5.73 \pm 2.46$ & $5.76 \pm 2.37$ \\
\hline$\overline{A R I(t)}$ & $4.88 \pm 1.47^{\S}$ & $4.90 \pm 1.61^{\#}$ & $5.30 \pm 1.52$ & $5.42 \pm 1.61$ \\
\hline intra-subject variability & $2.44 \pm 0.59$ & $2.24 \pm 0.65$ & $2.51 \pm 0.75$ & $2.32 \pm 0.80$ \\
\hline coefficient of variation $(\%)$ & $54.3 \pm 19.0$ & $52.4 \pm 25.7$ & $53.4 \pm 27.2$ & $48.9 \pm 26.6$ \\
\hline
\end{tabular}

$\S p<0.05$ in relation to the Finapres (right MCA)

${ }^{\#} \mathrm{p}<0.01$ in relation to the Finapres (left MCA) 
Figure 1. Influence of velocity signal-to-noise ratio on simulations with fixed values of ARI. A. $\mathrm{ARI}_{\mathrm{AO}}$ bias (in units of $\mathrm{ARI}$ ) in comparison with reference values of $\mathrm{ARI}$ for $\mathrm{ARI}=0$ (circles), 5 (squares) and 9 (triangles), respectively. B. Coefficient of variation of intrasubject variability. Error bars represent \pm 1 SEM.

Figure 2. Influence of ARI on estimated intra-subject mean values of ARI (top) and variability (bottom) in simulations with fixed value of $A R I$ (Reference $A R I)$ for $S_{N}=3 d B$. Continuous ARI estimates were derived with the Finapres (black columns) or intra-aortic BP (grey columns) recordings from 27 subjects. The error bars represent \pm 1 SD.

Figure 3. A. Representative estimate of continuous ARI for simulation of time-varying staircase ARI. The mean aortic BP for this subject was used with the staircase changes in ARI to generate the CBFV signal shown at the top. The ARI(t) estimates at the bottom were derived from $\mathrm{BP}_{\mathrm{AO}}$ (solid line) and $\mathrm{BP}_{\mathrm{FIN}}$ (dotted line). B. Population mean values $(\mathrm{N}=27)$ of continuous ARI for estimates obtained with mean aortic BP (solid line) and the Finapres (dotted line). The moving window duration for estimating the ARI was $60 \mathrm{~s}$ and the CBFV $\mathrm{SNR}_{\mathrm{V}}$ was $3 \mathrm{~dB}$. The original staircase time-varying ARI signal is shown by the broken line. The error bars represent the largest \pm 1 SD in each case.

Figure 4. Time-series of $\mathrm{BP}_{\mathrm{AO}}(\mathrm{A}), \mathrm{BP}_{\mathrm{FIN}}(\mathrm{B})$, right (C) and left (D) $\mathrm{CBFV}$ and right (E) and left $(\mathrm{F})$ estimates of $A R I(t)$ in a 58 year old male subject derived from $\mathrm{BP}_{\mathrm{AO}}$ (solid line) and $\mathrm{BP}_{\mathrm{FIN}}$ (interrupted line).

Figure 5. Population cumulative probability distributions of $A R I(t)$. A. Simulations of fixed values of $A R I$ for $S_{N R}=3 \mathrm{~dB}$. B. Distributions obtained with patient data for $\mathrm{ARI}_{\mathrm{FIN}}$ (circles) and $\mathrm{ARI}_{\mathrm{AO}}$ (triangles), for both right (closed symbols) and left (open symbols) MCA. 

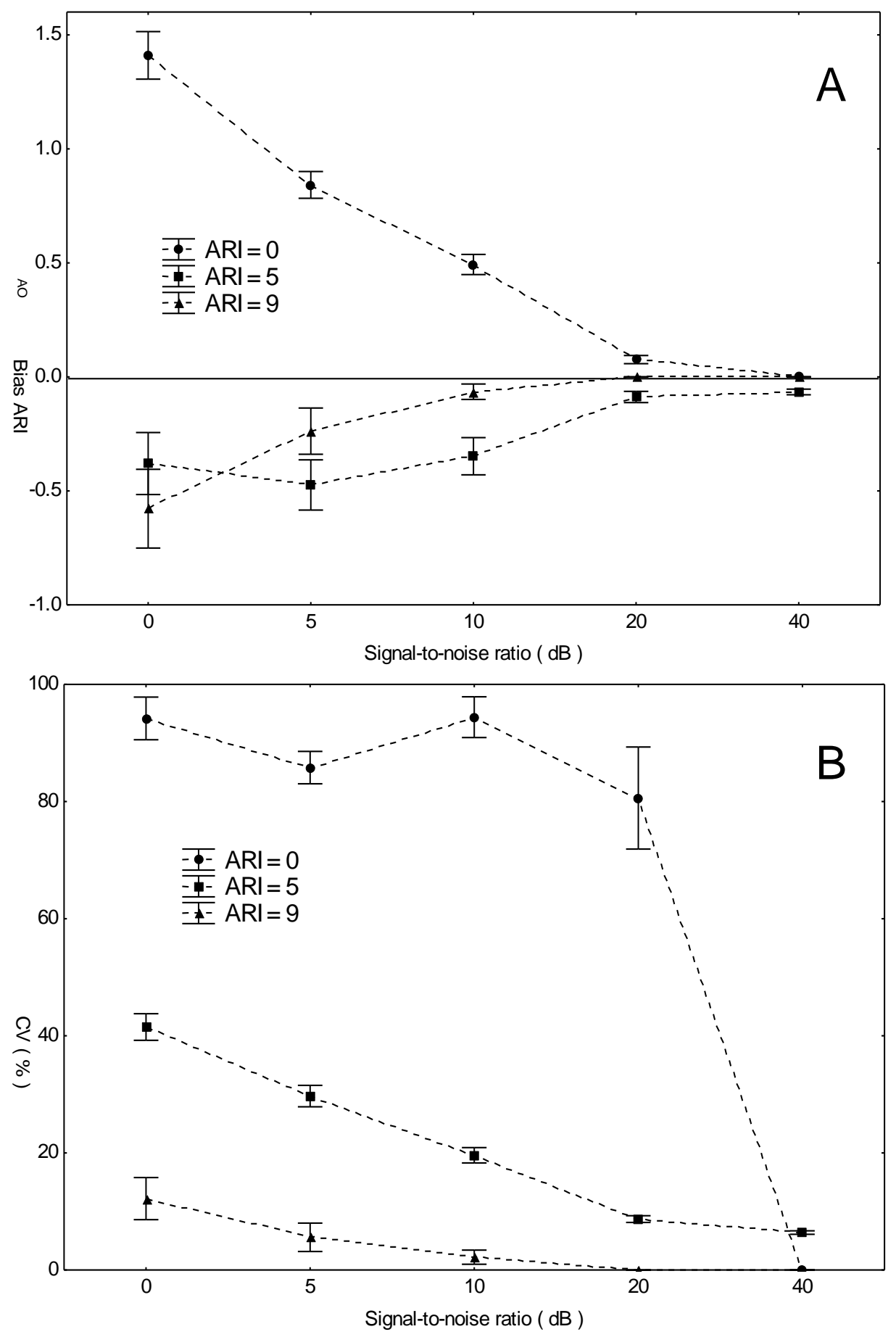

Figure 1 

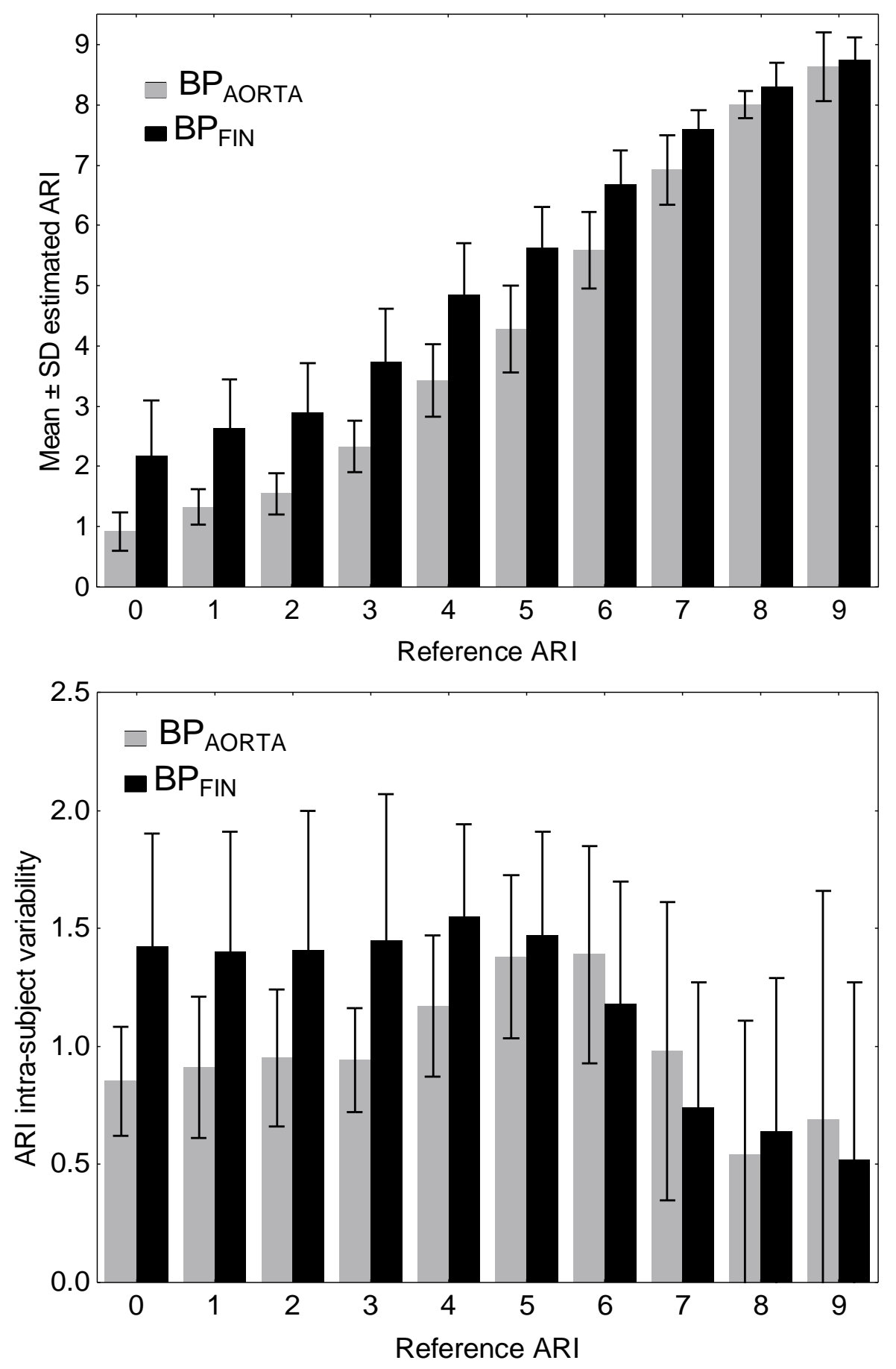

Figure 2 


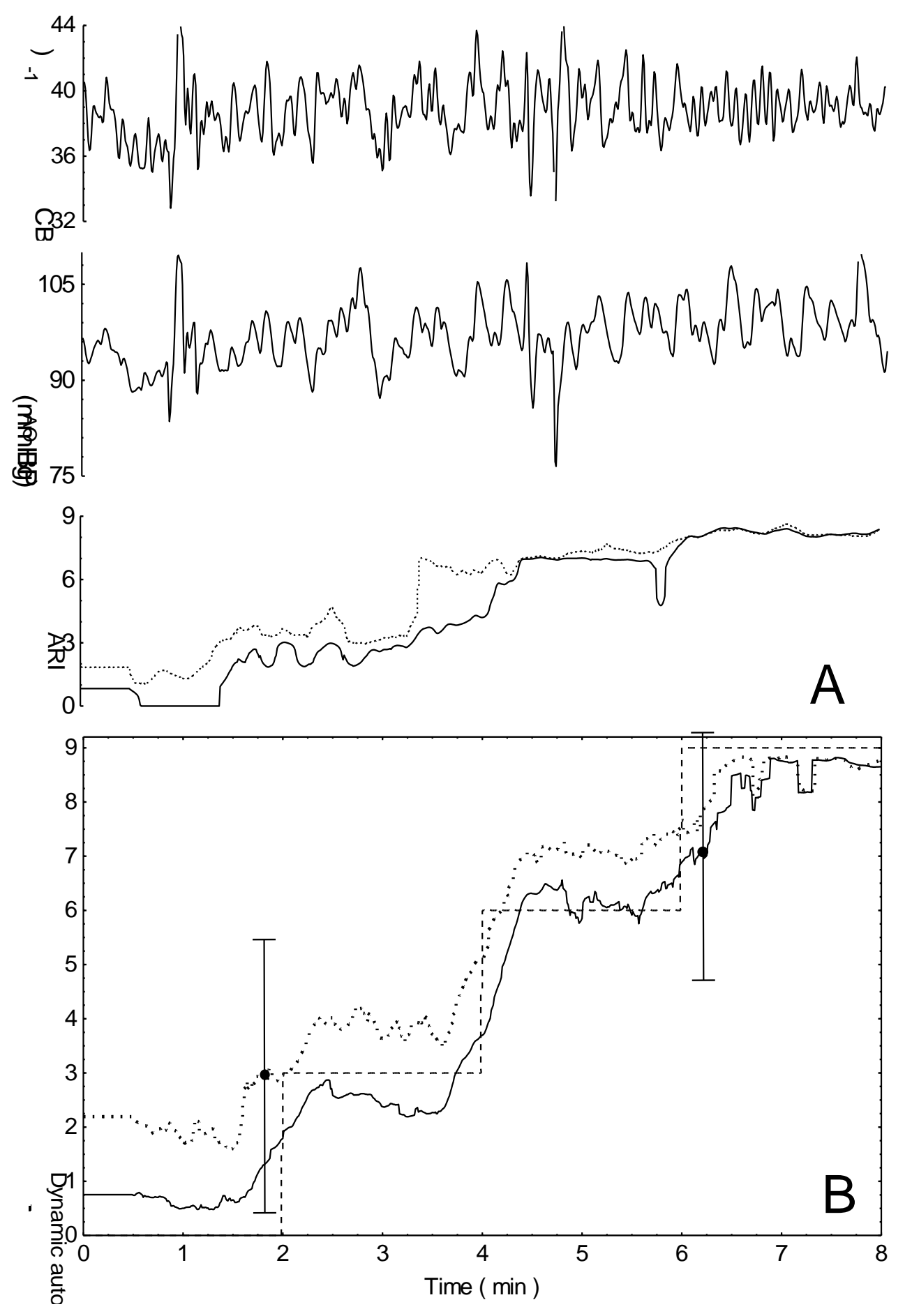

Figure 3 


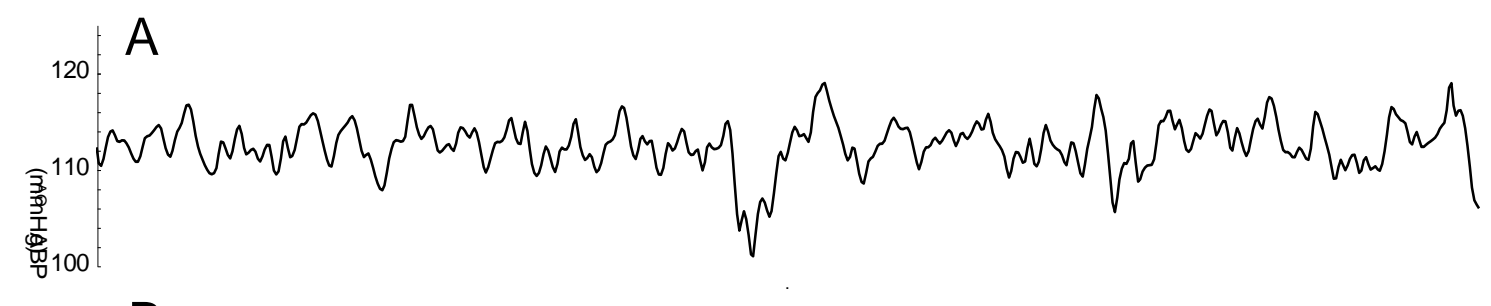

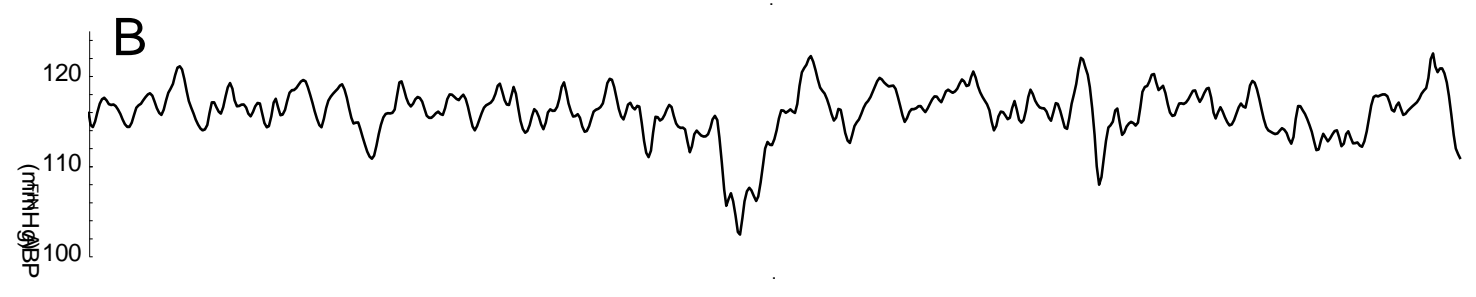

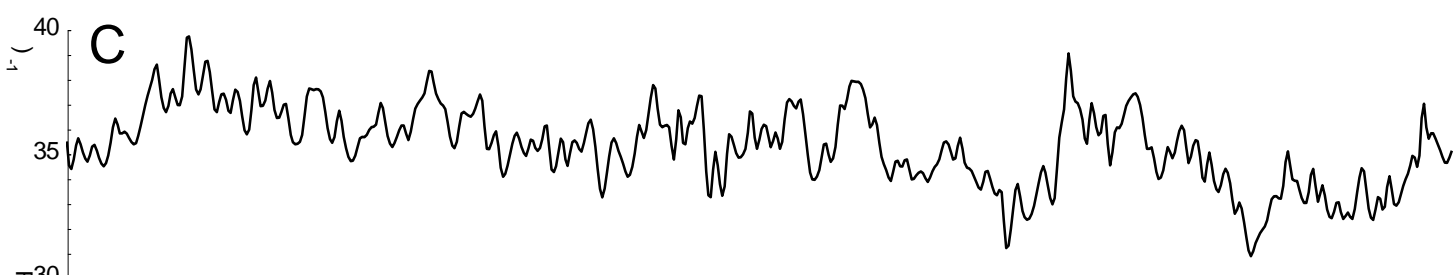

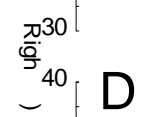

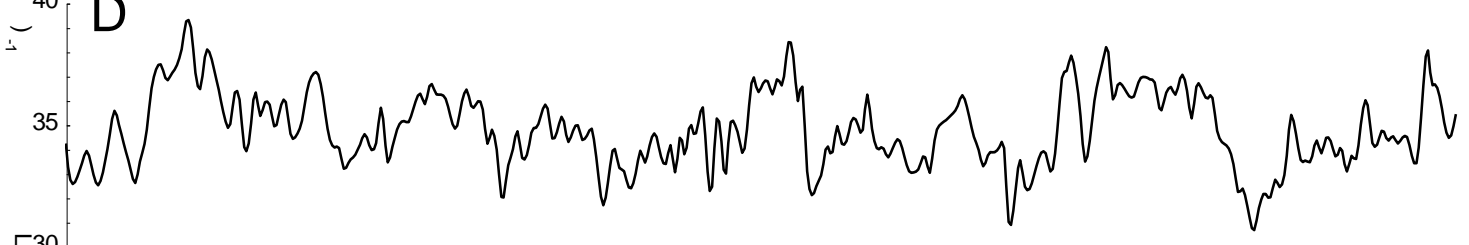
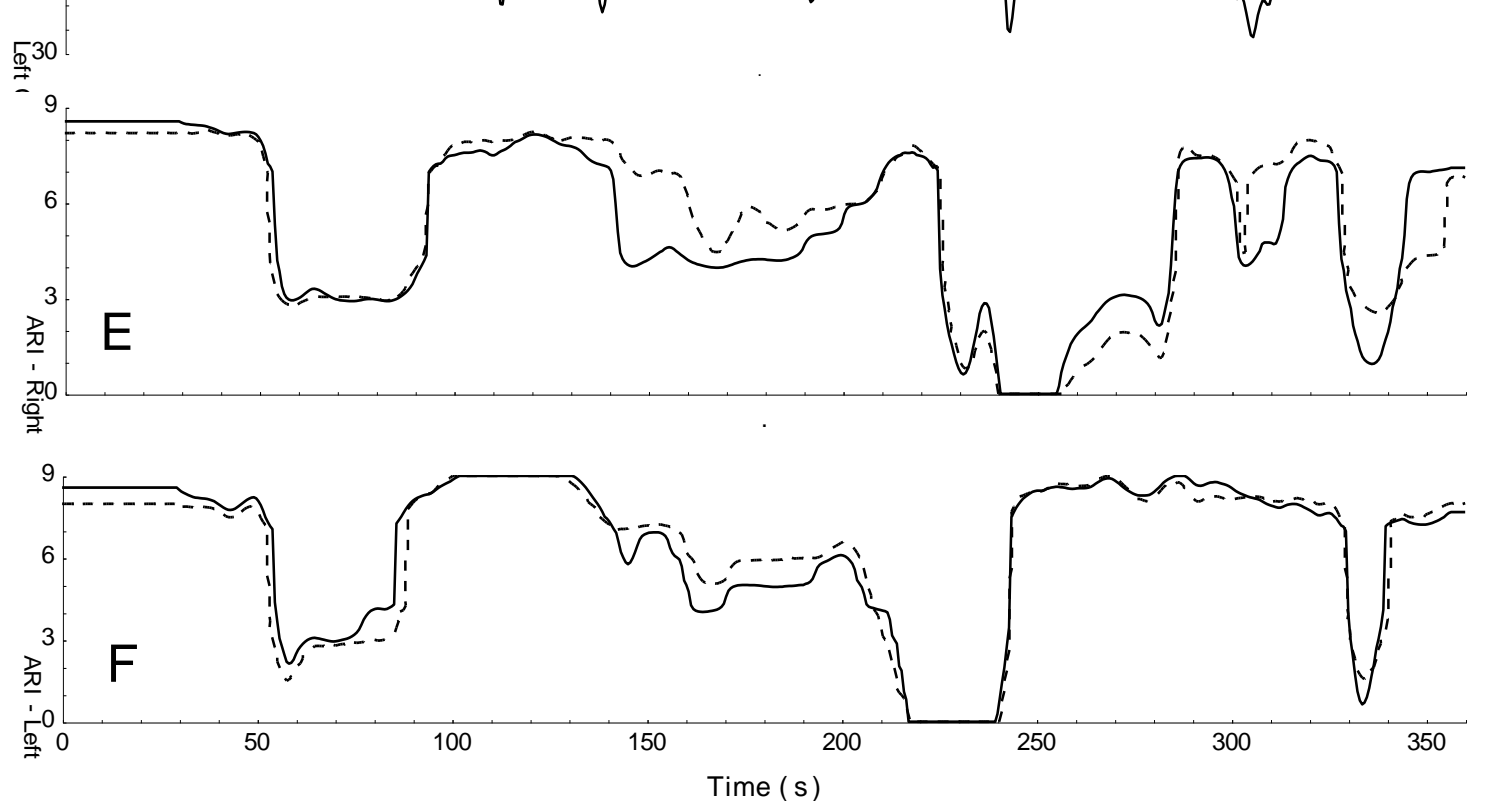

Figure 4 

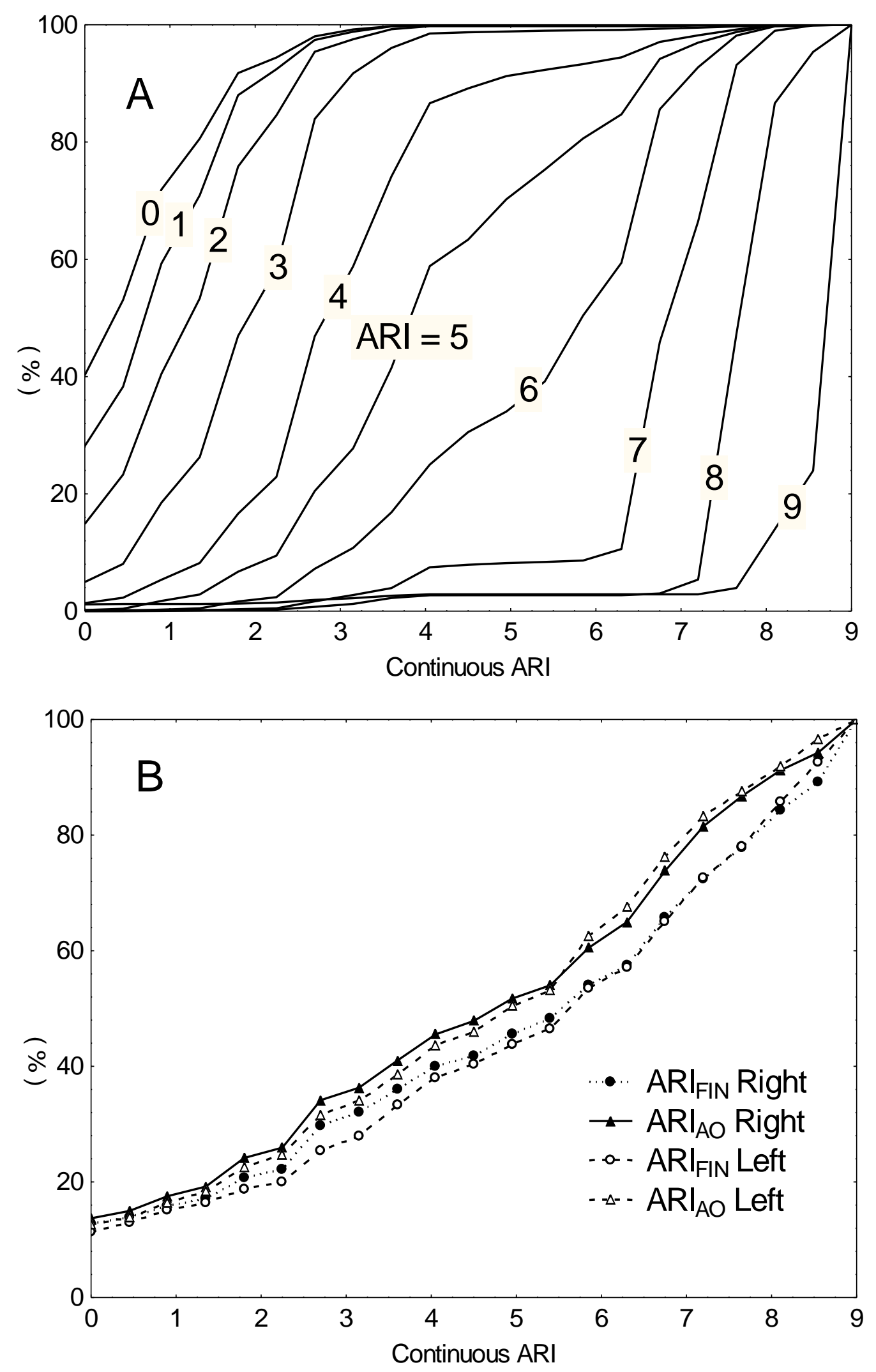

Figure 5 